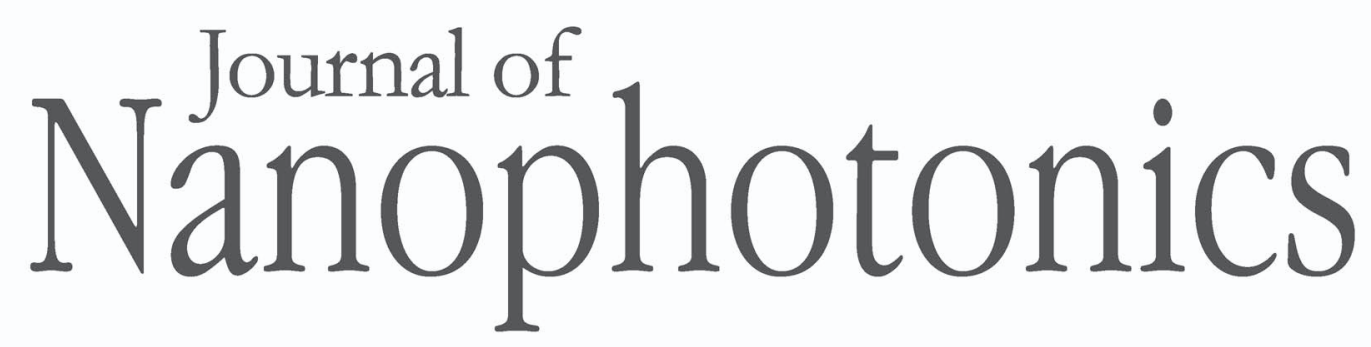

SPIEDigitalLibrary.org/jnp

\title{
Low-energy single-optical-cycle soliton self-compression in air-silica nanowires
}

\author{
Amine Ben Salem \\ Rim Cherif \\ Mourad Zghal
}




\title{
Low-energy single-optical-cycle soliton self-compression in air-silica nanowires
}

\author{
Amine Ben Salem, Rim Cherif, and Mourad Zghal \\ University of Carthage, Engineering School of Communication of Tunis (Sup'Com), \\ Cirta'Com Laboratory, Ghazala Technopark, 2083, Ariana, Tunisia \\ rim.cherif@supcom.rnu.tn
}

\begin{abstract}
We investigated and optimized the process of soliton self-compression in few millimeters-long air-silica nanowires. A 100 fs prechirped input pulse was compressed to a $1.4 \mathrm{fs}$ pulse by pumping at very low energy of $2.5 \mathrm{~nJ}$ an air-silica nanowire. More than one octave spanning coherent broadband supercontinuum extending from 260 to $1800 \mathrm{~nm}$ was generated. @ 2011 Society of Photo-Optical Instrumentation Engineers (SPIE). [DOI: 10.1117/1.3662888]
\end{abstract}

Keywords: ultrafast nonlinear optics; soliton self-compression; air-silica nanowire; supercontinuum generation; coherence.

Paper 11092LR received Sep. 16, 2011; revised manuscript received Oct. 29, 2011; accepted for publication Oct. 31, 2011; published online Nov. 16, 2011.

\section{Introduction}

Air-silica nanowires with core diameters smaller than the wavelength have attracted considerable interest due to their unique dispersion profiles and nonlinear properties for wide range of applications. ${ }^{1,2}$ These nanowires are not only suitable for nanophotonic devices but also enable soliton self-compression (SSC), dispersion compensation, and broadband supercontinuum (SC) generation. ${ }^{3,4}$ Much effort has been dedicated in generating pulses in the few-optical-cycle regime which has permitted important achievements in diverse fields such as the study of ultrafast dynamics in matter, high-order harmonic generation, extreme nonlinear optics, and attosecond physics. ${ }^{5,6}$ An impressive approach toward the monocycle regime dating back to the $1980 \mathrm{~s}$ has been used to generate compressed pulses relying on the concept of SSC. ${ }^{7}$ The introduction of highly nonlinear photonic nanowires with anomalous group velocity dispersion at visible and near-infrared wavelengths has enormously contributed in the study of SSC techniques and the generation of few to single-optical cycles. The results approaching the single-cycle regime have been reported by Foster et al. using tapered air-silica nanowires. ${ }^{8}$ They experimentally demonstrated the SSC of 70 to $6.8 \mathrm{fs}$ compressed pulse by injecting light into a 2-mm long $980 \mathrm{~nm}$ core diameter air-silica nanowire.

In this paper, we demonstrate the generation of a compressed pulse as short as $1.4 \mathrm{fs}$ (ca. 0.52 single-optical-cycle) in a 1.15 - $\mathrm{mm}$ long $800 \mathrm{~nm}$ core-diameter air-silica nanowire acting as a dispersion compensating fiber. Therefore, to the best of our knowledge, this is the shortest pulse and the highest compression ratio ever achieved and reported in air-silica nanowires at a low energy level. First, a full modal analysis of the optical properties of air-silica nanowires including chromatic dispersion, effective mode area, and nonlinear coefficient is presented. Investigation of the evanescent field has been conducted and the cut-off condition of single mode operation in air-silica nanowires is evaluated. Secondly, the optimization of the SSC, by pumping at very low pulse energy of $2.5 \mathrm{~nJ}$, a $100 \mathrm{fs}$ prechirped input pulse in the $800 \mathrm{~nm}$ air-silica nanowire is achieved. Moreover, more than one octave spanning coherent SC is generated extending from 260 to $1800 \mathrm{~nm}$.

$1934-2608 / 2011 / \$ 25.00$ @ 2011 SPIE 


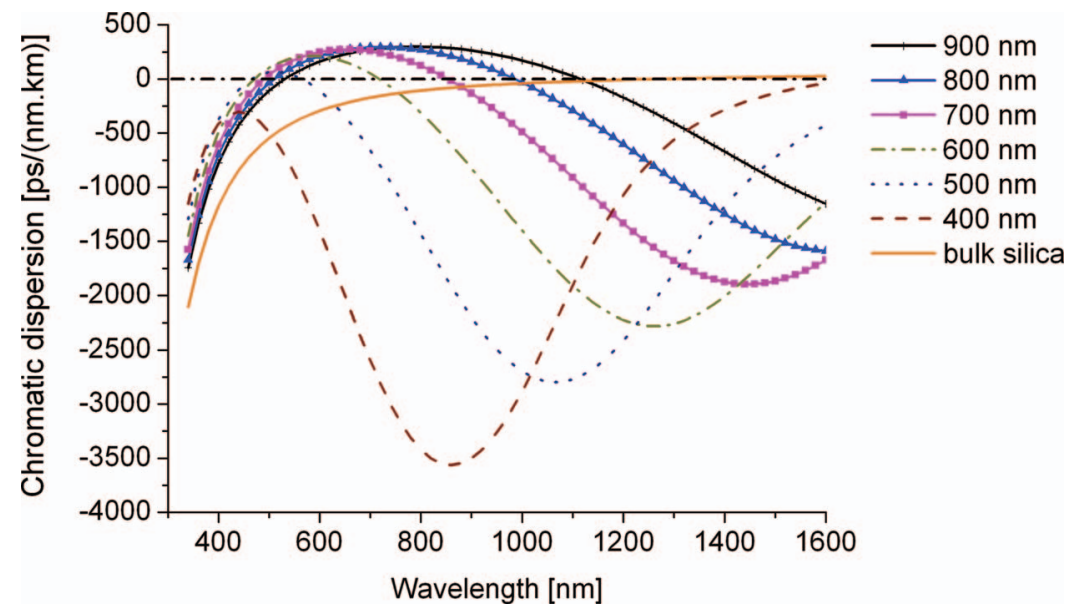

Fig. 1 Calculated chromatic dispersion of air-silica nanowires with core diameters ranging from 400 to $900 \mathrm{~nm}$.

\section{Optical Properties Modeling}

The optical properties of the air-silica nanowires are calculated using the glass-rod-in-air model. ${ }^{9}$ With this model, we analyze the confinement of the fundamental mode, the evolution of the dispersion as a function of the wavelength, the effective area, and the nonlinear coefficient. A full vectorial finite element method with dense meshes made up of $10^{4}$ to $10^{5}$ is developed to calculate the propagation constant $\beta$ of the fundamental mode $\mathrm{HE}_{11}$. By expanding the modepropagation constant $\beta$ in a Taylor series at a central frequency $\omega_{0}$, the chromatic dispersion can be determined through the group velocity dispersion (GVD) parameter $\beta_{2}$ as a function of the wavelength. ${ }^{10}$ We calculate the chromatic dispersion as a function of the wavelength for different core diameter air-silica nanowires ranging from 400 to $900 \mathrm{~nm}$, as seen in Fig. 1. Good agreement is found with chromatic dispersions calculated in Refs. 4 and 8.

By reducing the nanowire's core diameter, the region of the anomalous GVD exhibited between two zero dispersion wavelengths can be shifted toward the blue wavelength region. This property is a result from the dominance of the waveguide dispersion over the material dispersion (bulk silica) for air-silica nanowires. With the appropriate choice of the core size, the overall GVD can be highly engineered and the second zero dispersion wavelength can be positioned near the pump wavelength to yield anomalous, normal, or zero GVD. We notice that the largest region of the anomalous GVD centered around a pump wavelength of $\lambda_{p}$ $=800 \mathrm{~nm}$ and accompanied with low third-order dispersion $\left(\beta_{3}\right)$ is found for the $800 \mathrm{~nm}$ corediameter air-silica nanowire $\left(\beta_{2}=-9.12 \times 10^{-2} \mathrm{ps}^{2} \mathrm{~m}^{-1}\right.$ and $\left.\beta_{3}=-5.28 \times 10^{-6} \mathrm{ps}^{3} \mathrm{~m}^{-1}\right)$. This property is primordial to achieve efficient SSC which results from the interplay between the dispersion and the self-phase modulation effects. This fact is due to exotic dispersion profiles and high nonlinearities provided by air-silica nanowires. Thus, the $800 \mathrm{~nm}$ core-diameter air-silica nanowire is selected to optimize the SSC at $\lambda_{p}=800 \mathrm{~nm}$.

By calculating the longitudinal component of the Poynting vector $S_{z}$ for the sub-wavelength nanowire structures, a significant fraction of the power is found to reside in the evanescent field. This evanescent field interacts directly with the cladding (air)-glass interfaces. The calculation of the Poynting vector shows that when the optical wavelength $\lambda$ is larger than the nanowire's core diameter $d$, the evanescent field starts to dominate and its fractional power increases. Although the modeling has been conducted for a fixed $\lambda$ and varying $d$, one could assume that the behavior of the evanescent field and its fractional power remain the same as long as the ratio $d / \lambda$ does not change. This is justified by the small changes $\Delta n / n \approx 0.02$ of the refractive index of silica glass in a large wavelength range between 400 and $1800 \mathrm{~nm}$. To obtain straightforward information 


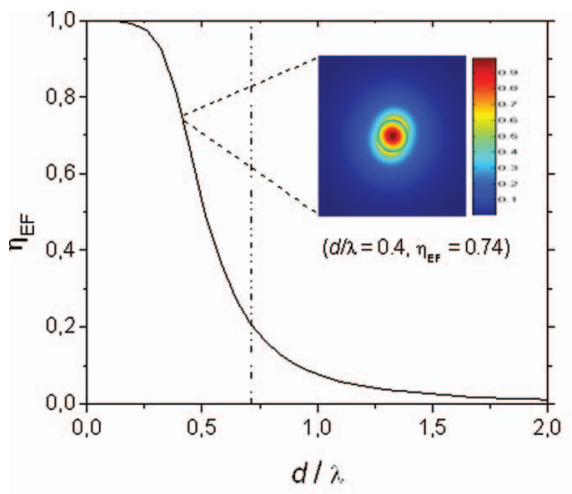

(a)

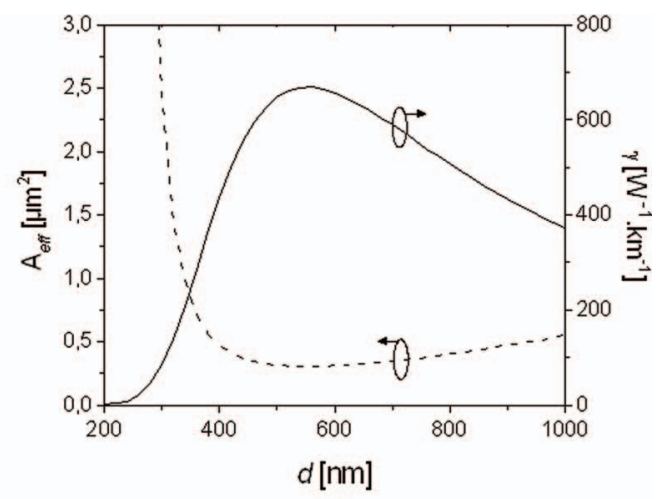

(b)

Fig. 2 (a) Fractional power $\eta_{\mathrm{EF}}$ outside the core as a function of the normalized diameter $d / \lambda$. (b) Effective mode area $\left(A_{\text {eff }}\right)$ and nonlinear coefficient $(\gamma)$ of air-silica nanowires for different core diameters at $\lambda_{p}=800 \mathrm{~nm}$.

about the power distribution in the radial direction, we evaluate the fractional power $\eta_{\mathrm{EF}}$ outside the core which is given in Ref. 9.

Figure 2(a) shows the fraction of power of the fundamental mode propagating outside the core as a function of the normalized diameter $d / \lambda$. We notice that by increasing $d / \lambda$, the evanescent field decreases until reaching a value of $9 \%$ for a ratio $d / \lambda=1$ and almost $1 \%$ for a ratio of $d / \lambda=2$. Therefore, with a careful design of air-silica nanowire with a core diameter $d<0.25 \lambda$, more than $98 \%$ of the light can propagate outside the core. The evanescent field surrounding the nanowire and the strong radial confinement of the light make air-silica nanowires well-suited for an efficient and controlled interaction of guided light with matter and perfect devices for sensing applications. ${ }^{11}$ Like conventional optical fibers, air-silica nanowires experience singlemode $\left(\mathrm{HE}_{11}\right)$ guiding for $V<2.405 .^{9}$ The dashed line in Fig. 2(a) indicates the cut-off condition for single mode operation. We clearly see that only the fundamental mode propagates when $d$ $<0.71 \lambda$ (i.e., $V<2.405$ ). At this cut-off condition, the fractional power inside the core is equal to $80 \%$ of the total propagating light. For instance, at $\lambda_{p}=800 \mathrm{~nm}$, the critical core diameter for single mode operation is $d_{\mathrm{SM}}=568 \mathrm{~nm}$. Since the air-silica nanowire exhibits a significant fraction of the power propagating outside the core, an accurate estimation of the nonlinear coefficient $\gamma$ is needed. ${ }^{12}$ Figure 2(b) depicts the effective mode area $A_{\text {eff }}=2 \pi n_{2} /\left(\lambda_{p} \gamma\right)$ and $\gamma$ calculated at $\lambda_{p}=800 \mathrm{~nm}$ for air-silica nanowires with core diameters ranging from 200 to $1000 \mathrm{~nm}$. It illustrates that the mode confinement determined by the effective mode area becomes stronger when reducing the core diameter of the air-silica nanowire. This behavior evolves linearly and reaches a minimum effective mode area (maximum nonlinear coefficient) value for a core diameter of about $550 \mathrm{~nm}$. A high nonlinear coefficient of $507 \mathrm{~W}^{-1} \mathrm{~km}^{-1}$ is calculated for the $800 \mathrm{~nm}$ air-silica nanowire by using $n_{2}=2.6 \times 10^{-20} \mathrm{~m}^{2} \mathrm{~W}^{-1} \cdot{ }^{10}$

\section{Soliton Self-compression and Supercontinuum Generation}

The investigation of the nonlinear propagation is based on the resolution of the generalized nonlinear Schrödinger equation (GNLSE) which takes into account the contribution of linear and nonlinear effects and describes the temporal and the longitudinal dependence of the pulse envelope $A(z, t) .{ }^{10}$ The resolution of the GNLSE is performed using the symmetrized split step Fourier method. ${ }^{13}$ We set the loss coefficient to $1 \mathrm{dBm}^{-1}$ (Ref. 4) and used an accurate model including the full dispersion profile. We consider the propagation of an $N$ 'th order soliton having the envelope field expression $A(0, t)=\sqrt{P_{0}} \sec h\left(t / T_{0}\right) \exp \left(-i C \cdot t^{2} / 2 T_{0}^{2}\right)$, where $P_{0}$ is the peak power and $T_{0}$ is the input soliton duration defined as $T_{\mathrm{FWHM}} / 1.763 . T_{\mathrm{FWHM}}$ is the 

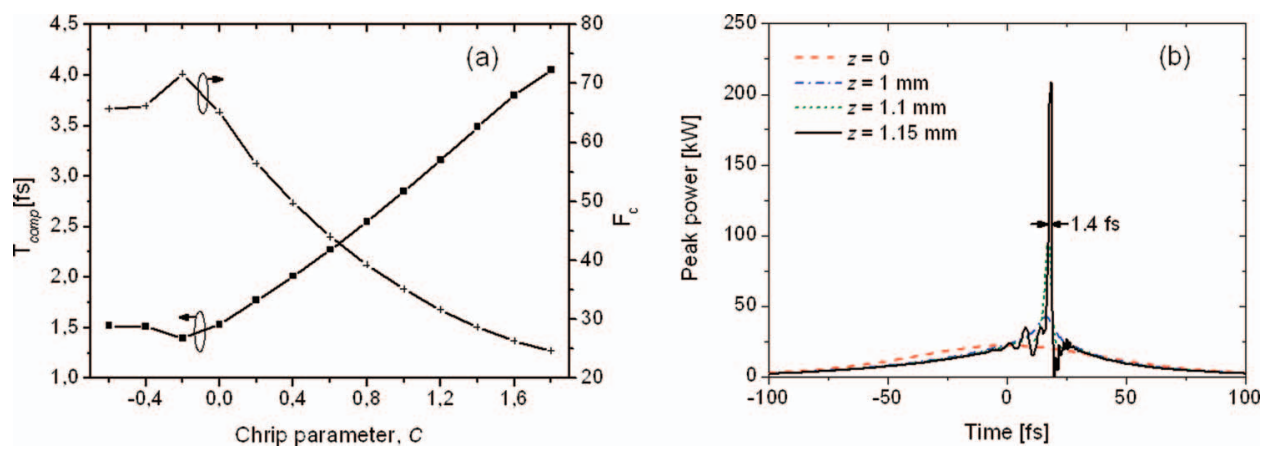

Fig. 3 (a) Effect of the initial chirp on the compression efficiency. (b) Temporal evolution of 100 fs prechirped input as a function of the propagation distance at $\lambda_{p}=800 \mathrm{~nm}$.

input pulse full width at half maximum (FWHM) duration. $C$ is the chirp parameter controlling the initial chirp. ${ }^{10}$

Our numerical study shows that input pulses with durations shorter than 100 fs with few nanojoule energy requires an optimal nanowire length considerably shorter than $1 \mathrm{~mm}$, which immediately poses a practical problem since it is difficult to obtain such short fiber lengths. However, enlarging the initial pulse duration at the same low input energy allows the increase of the nanowire length so that $100 \mathrm{fs}$ input pulse is found to overcome this difficulty. Air-silica nanowires' lengths on the order of $1 \mathrm{~mm}$ are then required, which can be easily fabricated as demonstrated in Ref. 8. Thus, in our calculations, the input pulse duration is taken as $T_{\text {FWHM }}$ $=100 \mathrm{fs}$.

In order to characterize the efficiency of the SSC, three parameters are introduced and denoted: 1. $z_{\mathrm{opt}}$ is the optimal length at which a maximum compressed pulse can be extracted with highest output preserved soliton pulse shape. 2. $F_{c}=T_{\mathrm{FWHM}} / T_{\text {comp }}$ is the compression factor defined as the ratio of the FWHM pulse durations at the input ( $\left.T_{\mathrm{FWHM}}\right)$ and the output of the nanowire $\left(T_{\text {comp }}\right)$. 3. $Q_{c}=P_{\text {comp }} /\left(P_{0} F_{c}\right)$ is the quality factor defined as the ratio of the peak power $\left(P_{\text {comp }}\right)$ of the compressed pulse with the input peak power $P_{0}$ and $F_{c}$. We start our analysis with the optimization of laser-pulse parameters (i.e., pulse duration, pulse shape, and chirp) on SSC and the spectral properties of the generated SC. We first examine the effect of the chirp on the compression factor. To this end, we simulate an initial nonchirped hyperbolic-secant pulse and vary the input pulse energy. A maximum compressed pulse with duration of $1.53 \mathrm{fs}$ in a $1.15 \mathrm{~mm}$ nanowire length is found with an input energy of $2.5 \mathrm{~nJ}$. Then, we vary the input chirp $C$ and we analyze the nonlinear evolution aiming to extract the optimal chirp value giving the maximum compressed pulse.

Figure 3(a) shows the effect of the variation of the chirp on the duration of the final compressed pulse $T_{\text {comp. }}$. We find that positive chirp degrades the temporal compression while negative chirp enhances the SSC. The optimal chirp value of $C=-0.2$ is found to maximize the temporal compression. In conclusion, we find that a very low input energy of $2.5 \mathrm{~nJ}$ prechirped input hyperbolic-secant pulse at a pump wavelength of $800 \mathrm{~nm}$ gives an optimum temporal compressed pulse. Figure 3(b) shows the result of the optimization of the temporal evolution of the chirped hyperbolic-secant pulse with $C=-0.2$ as a function of the propagation distance. We extract a maximum compressed pulse as short as 1.4 fs generated after $1.15-\mathrm{mm}$ long 800 $\mathrm{nm}$ core-diameter air-silica nanowire corresponding to the excitation of an input soliton order $N=19.8$. This interaction corresponds to a very high compression factor $F_{c}=71.43$ and a quality factor $Q_{c}=0.13$. We notice that by overpassing the optimal propagation distance $\left(z_{\mathrm{opt}}\right.$ $=1.15 \mathrm{~mm}$ ), the temporal pulse profile presents a remarkable increasing multipeak and broadened structure due to soliton fission induced by Raman scattering and high-order dispersions. The main aim of the analysis presented in this section is to understand the spatiotemporal dynamics behind the supercontinuum SSC to few-cycle durations. 
(a)
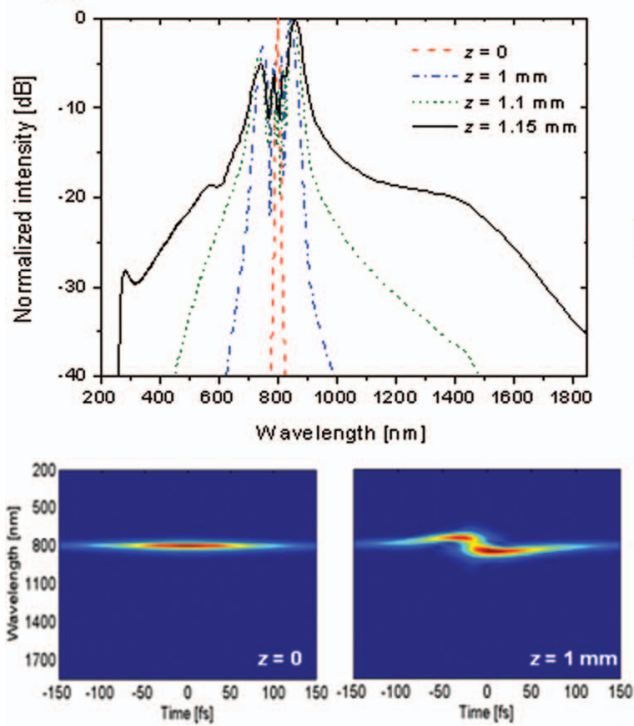

(c)

(b)
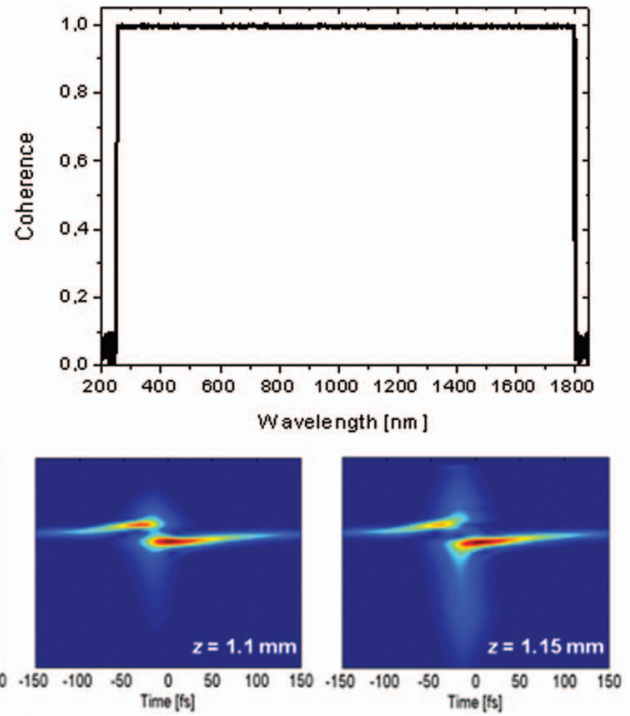

Fig. 4 (a) Spectral evolution of 100 fs input pulse with $2.5 \mathrm{~nJ}$ input as a function of the propagation distance $z$. (b) Degree of coherence of the generated SC. (c) Spectrogram representation of the pulse at each propagation distance.

Figure 4(a) shows the spectral evolution of $100 \mathrm{fs}$ input pulse with $2.5 \mathrm{~nJ}$ input energy in the $800 \mathrm{~nm}$ core-diameter air-silica nanowire as a function of the propagation distance until the optimal SSC is achieved. The analysis of the coherence properties of the generated SC with adding one photon per mode noise with a random phase ${ }^{14}$ shows from an ensemble average of 60 independent simulations that the generated SC is perfectly coherent over the entire generated bandwidth [see Fig. 4(b)]. The corresponding spectrogram representations are shown at each of the propagation distances in Fig. 4(c). Broadband SC from ultraviolet to mid-infrared regions extending from 260 to $1800 \mathrm{~nm}$ is generated.

These features reflect the growth of the symmetrical broadening when the propagation distance increases to $1 \mathrm{~mm}$. This confirms that the effect of self-phase modulation is the predominant effect given such broadening. However, an asymmetric spectral profile starts to appear toward infrared wavelengths and is observed at the optimal length. This is justified by the fact that the compressed pulse undergoes soliton fission caused by the Raman effect but with very low intensity [as seen in Fig. 3(b)] so that Raman solitons are generated and the SC is redshifted. Although very small pulse fluctuations and peaks appear around the highest peak of the compressed pulse, the extraction of the optimal compressed pulse is still valid since a large intensity gap is depicted between the highest and smallest peaks. Comparable broadband compressible SC was theoretically generated in the $800 \mathrm{~nm}$ nanowire by Foster et al., ${ }^{8}$ but in a very short nanowire length of $650 \mu \mathrm{m}$ which can be considered as a practical problem to obtain such a short fiber length. Thus, the idea of enlarging the initial pulse to $100 \mathrm{fs}$ is proposed with an implemented prechirping technique and generating efficient compressible SC to a single-cycle pulse is achieved.

\section{Conclusion}

Soliton self-compression has been investigated in an $800 \mathrm{~nm}$ air-silica nanowire. A $1.4 \mathrm{fs}$ compressed pulse starting from $100 \mathrm{fs}$ is obtained at a very low input pulse energy of $2.5 \mathrm{~nJ}$ in only a $1.15-\mathrm{mm}$ long nanowire. Thus, a very high compression factor of 71.43 is achieved. 
The generation of more than one octave spanning coherent SC from UV to near-IR is shown. Air-silica nanowires are found to be very promising for designing white light coherent sources and suitable waveguides for dispersion compensation and high SSC which opens new horizon toward extreme nonlinear optics and attosecond physics.

\section{Acknowledgments}

We acknowledge partial support of "Institut Télécom" through the "Futur et Ruptures" $\mathrm{PhD}$ thesis of A. Ben Salem. We thank John Dudley from Institut Femto-ST, University of FrancheComté, Besançon, France for collaboration and useful scientific discussions.

\section{References}

1. L. M. Tong, R. R. Gattass, J. B. Ashcom, S. L. He, J. Y. Lou, M. Y. Shen, I. Maxwell, and E. Mazur, "Subwavelength-diameter silica wires for low-loss optical wave guiding," Nature 426, 816-819 (2003).

2. M. Foster, C. Turner, M. Lipson, and A. L. Gaeta, "Nonlinear optics in photonic nanowires," Opt. Express 16, 1300-1320 (2008).

3. R. R. Gattass, G. T. Svacha, L. M. Tong, and E. Mazur, "Supercontinuum generation in submicrometer diameter silica fibers," Opt. Express 14, 9408-9414 (2006).

4. S. G. Leon-Saval, T. A. Birks, W. J. Wadsworth, P. S. J. Russell, and M. W. Mason, "Supercontinuum generation in submicron fibre waveguides," Opt. Express 12, 2864-2869 (2004).

5. T. Brabec and F. Krausz, "Intense few-cycle laser fields: Frontiers of nonlinear optics," Rev. Mod. Phys. 72, 545-591 (2000).

6. F. X. Kartner, Ed., Few-Cycle Laser Pulse Generation and Its Applications, Springer Verlag, Berlin, Germany (2004).

7. L. F. Mollenauer, R. H. Stolen, J. P. Gordon, and W. J. Tomlinson, "Extreme picosecond pulse narrowing by means of soliton effect in single-mode optical fibers," Opt. Lett. 8, 289-291 (1983).

8. M. Foster, A. L. Gaeta, Q. Caoand, and R. Trebino, "Soliton-effect compression of supercontinuum to few-cycle durations in photonic nanowires," Opt. Express 13, 6848-6855 (2005).

9. L. Tong, J. Lou, and E. Mazur, "Single-mode guiding properties of subwavelength-diameter silica and silicon wire waveguides," Opt. Express 12, 1025-1035 (2004).

10. G. P. Agrawal, Nonlinear Fiber Optics, 4th Ed., Academic, Elsevier, San Diego, USA (2006).

11. G. Brambilla, "Optical fibre nanotaper sensors," Opt. Fiber Tech. 16, 331-342 (2010).

12. M. A. Foster, K. D. Moll, and A. L. Gaeta, "Optimal waveguide dimensions for nonlinear interactions," Opt. Express 12, 2880-2887 (2004).

13. R. Cherif, M. Zghal, L. Tartara, V. Degiorgio, "Single-sided supercontinuum generation in a photonic crystal fiber by selective excitation of the third-order mode," Proc. SPIE 6990, 699000 (2008).

14. J. M. Dudley and S. Coen, "Coherence properties of supercontinuum spectra generated in photonic crystal fiber and tapered optical fiber," Opt. Lett. 27, 1180-1182 (2002). 